\title{
MEDIA COMPETENCE AS A FACTOR OF PROFESSIONAL SELF-REALIZATION OF A PRACTICAL PSYCHOLOGIST
}

\section{Vsevolod Zelenin ${ }^{1}$ \\ Tetiana Ponomarenko}

DOI: https://doi.org/10.30525/978-9934-588-15-0-122

Abstract. The purpose of the article is to explore the specifics and necessary skills for the successful activity of future psychologists in the media space. The method of content analysis allows us to determine the types, specificities, and peculiarities of media activity of psychologists; to establish the necessary skills that should be developed by future specialists; identify factors that adversely affect their professional formation. Method. This research presented two samples of a total of 163 individuals: practicing psychologists $(n=103)$ and fourth-year psychology students $(n=60)$. Using content analysis, we explored the specifics and skills that psychologists need to effectively pursue the professional activity in the media space. V. Ryakhovsky Test and the M. Snyder Test help us to establish the level of communicative competence of psychologists and psychology students. With the help of the C. Spielberger Scale, we examined the level of psychologists and student anxiety. The results of the research showed that psychologists perform three activities in the media space: educational, advertising (SMM) and individual counseling. $80 \%$ have an education, which is one of the most important activities of a practical psychologist. Advertising of own products and psychological services is $15 \%$. Individual counseling is only $5 \%$. Educational activity of the psychologist in the media space solves the following tasks: 1). Creates true ideas about the range of professional tasks of the psychologist in the person's mind. 2). Forms a complex of knowledge for people to solve psychological problems (conflicts, difficult life situations and so on) independently. 3). Promoting healthy habits and healthy lifestyles. 4). Announcing interesting, useful and informative online and

${ }^{1}$ Candidate of Psychological Sciences, Associate Professor,

Department of Political Psychology and Social Legal Technologies,

National Pedagogical Dragomanov University, Ukraine

${ }^{2}$ Postgraduate Student at the Department of Political Psychology and Social Legal Technologies, National Pedagogical Dragomanov University, Ukraine

(C) Vsevolod Zelenin, Tetiana Ponomarenko 
offline events that take place in the country as a whole or in a specific city in order to enhance the psychological culture of the population. 5). Mental health preservation is the rapid informing of the population about a specific algorithm for actions in case of emergencies or rehabilitation after them. 6). Dissemination of information about useful and interesting discoveries of scientists, about achievements and practices in order to popularize psychological science and quickly bring new achievements to real consumers. Psychologists typically use the social networks Facebook, Instagram, Telegram, YouTube, and email and webinars to advertise their services and attract clients. And in individual work they prefer Skype, Messenger, Viber and WhatsApp. These three activities are needed a high level of communication competence. We have found that $45,6 \%$ of psychologists and $47,9 \%$ of future psychologists do not have a good level of communication skills and only $6,7 \%$ psychologists and $26,7 \%$ of students have a high level of anxiety. Practical implications. As the current market for psychological services requires the introduction of media into professional activity, it is important to understand what kind of knowledge and skills of media professionals future professionals need to possess. Value/originality. Assessment of the media activity of psychologists has allowed to determine the types and specifics of successful activity and to establish the knowledge and skills that should be formed in students-psychologists of higher education.

\section{Introduction}

Nowadays, modern innovative technologies form a huge part of everyone's life area. They open up new perspectives and determine the success of the professional activity of experts. Distance learning and online projects, which promote personal self-development, become very popular. In the last five years, psychologists have been actively using media tools in their professional activities. Media space opens up new possibilities and prospects for an expert. Consequently, professional activity has some advantages: convenience, saving of travel time, the ability to work remotely from any part of the world, expanding of the audience, expanding of business scope, social network advertising and others.

For the last twenty years, there have been significant changes in the provision of psychological services. Originally, it was only in-person counseling clients. Over time, telephone counseling, so-called "hot line, has gained 
popularity over the recent fifteen years. Nowadays, the Internet counseling takes its place. The research challenge concerns the fact that modern experts do not have the relevant knowledge and skills which are essential for successful professional activity in the media space. The goal of the article is to study the specifics and necessary skills for a successful activity and self-realization of future psychologists in the media space.

\section{Types of activity of a psychologist in the media space}

The authors mark three activity areas of a psychologist in the web space. $80 \%$ takes educational activity, which is one of the most important activities of a practical psychologist despite the area of his/her interests. It is presented in the entertainment format (short videos, interesting posts, useful stories, live streams) and designed to spread specific information about the special aspects, forms, types and ways of psychologists' activities.

In a broad sense, this type of activity is aimed at the psychological education of people because it acquaints them with the specifics and full range of activities of a practical psychologist, methods, opportunities, and a narrow understanding of educational activities covers the purposeful actions of psychologists, which are related to the dissemination of the necessary psychological knowledge. Media space is an important tool for educational activities: it allows not only to reach a large number of people but also to act quickly and accurately in situations of extreme urgency (prompt informing of the population about a particular operating procedure in case of a specific psychological threat).

Educational activity as the first work area of a psychologist in the media space completes the following tasks:

1) Generation of faithful ideas of the range of professional tasks of a psychologist in human consciousness. It is of key importance for residents of post-Soviet countries, who, unlike residents of EU and US countries, often have misconceptions about the psychologist's professional activity. In many cases, our fellow citizens refuse to employ psychology experts, even if it is necessary, confusing the activities of a psychologist, psychotherapist and psychiatrist. Sometimes they do not understand what problems or tasks can be addressed to them.

2) Formation of a complex of knowledge for the independent solution of psychological problems (conflicts, difficult life situations etc.). 
3) Promotion of healthy habits and lifestyle.

4) Announcement of interesting, useful and informative online and offline events which take place in the country or in a specific city in order to enhance the psychological culture of the population.

5) Preservation of mental health is a prompt informing of the population about a particular operating procedure in case of a specific psychological threat [4].

6) Dissemination of information about useful and interesting accomplishments of scientists, achievements and practices in order to popularize psychological science and familiarize real consumers with new advances promptly [6].

The second area, which covers about $15 \%$ of the activity of a psychologist on the Internet, is the promotion of own products and psychological services (SMM), that is, clients capture. Realising own educational activity, a psychologist becomes recognizable to the client and provides a certain level of confidence in himself as an expert, so he can advertise his products (books, checklists, marathons, trainings) and services (therapy, individual counseling or coaching sessions).

Thus, a third focus area emerges, that is, direct individual counseling. By applying media tools, the client first hears of a particular psychologist, gets acquainted with him and the specifics of his activity. The next stage forms a certain level of trust in the expert or vice versa and, if necessary, the client seeks individual counseling.

To advertise own services and to lure customers, psychologists usually use social networks Facebook, Instagram, Telegram, YouTube, mail out and webinars. In terms of face-to-face work, they prefer Skype, Messenger, Viber and WhatsApp.

Consequently, there is a need for a thorough study of educational and advertising trends because universities do not provide disciplines that would form the necessary knowledge and skills required for future psychologists. Professional activity at the media level allows the practical psychologist to improve the general level of psychological culture of the population, to expand the scope of his own psycho-business, to earn a high income and to achieve professional self-realization. Thus, that kind of professional activity requires new knowledge, skills and a high level of development of the communicative competence of psychologists. 


\section{Specifics of media activity of a practical psychologist}

The problems of the psychologist's activity in the media space were considered in the papers of foreign (A. Becker, D. Giles, V. Duke, V. Liaudis, R. McCall, S. Punching, G. Simon, S. Stoltz, Sh. Turkle et al.) and domestic (O. Adamenko, O. Arestova, V. Bondarovska, A. Hordieieva, V. Zelenin, I. Kotyk, O. Krutko, Yu. Maksymenko, L. Naidonova, L. Panchenko, N. Khmil, O. Shmielova et al.) scientists.

Using the content analysis, the authors determined the specific nature of the media activity of psychologists-practitioners of different profiles (consultants, coaches, therapists, art specialists, psychologists of institutions, etc.) with a total number of 103 persons. It was found that $59.2 \%$ of the respondents actively use modern media tools in their professional activities. They prefer Facebook and Instagram and rarely have their own YouTube channels and run webinars. This choice is caused by:

1) the specific nature of the above services as follows:

- Facebook users actively read texts [7, p. 74];

- Instagram followers are more attracted to visual content [5, p. 361];

- subscribers of YouTube channels are interested in the quality, innovative nature, originality and comprehensibility of video content $[5$, p. $77 ; 7$, p. 363];

- psychologists who conduct webinars should not be afraid of the camera, be fluent in the subject, have a high level of communicative competence [2, p. 3375].

2) specifics of targeted audience:

$-90 \%$ of Facebook audience is people over 18 years - the vast majority is $25-34$ years. These are people who have secondary or higher education and medium or high income [7, p. 79];

- Instagram audience is mostly young people aged 10-42 years, mostly female (beauty bloggers, moms who are on maternity leave, female psychologists) [5, p. 363];

- YouTube audience is more diverse as it contains a variety of content for both children and adults, both for women and men [5, p. 363; 7, p. 77].

The paper marks that only $20.3 \%$ of respondents use all options of Facebook. $38 \%$ actively involve new technologies in their professional practice, $16.7 \%$ almost do not use the network for their own professional activity, and $25 \%$ of respondents use Facebook as a personal account. 
The authors also pay attention to the functionality of communities' creation, which make it possible to develop a participatory culture of the psychological area, is hardly used (12.3\%).

The authors notice that active users (58.3\% of respondents) can be conditionally divided into three groups: those that use text content; those who use video content; those who use both video and text content. The authors interrogated 68 respondents (active users of media resources) and found that $50 \%$ (34 persons) prefer text communication due to underdeveloped level of communication competence and fears, as follows:

- fear of public speech;

- fear to lose the train of thoughts;

- fear of being at loss of words;

- fear of forgetting the text;

- fear of being ridiculed;

- fear of being criticized etc.

Thus, it is concluded that a modern expert should be involved in the media space with the purpose of professional self-realization. But the media activity of practical psychologists needs new skills and knowledge of the specifics of media tools $[3 ; 10]$ that is ensured by a high level of communicative competence [8], which, in turn, should be formed at the stage of school and university training.

\section{Research methodology}

The research was conducted from autumn 2018 - summer 2019. It involves two samples with a total of 163 individuals: practicing psychologists $(n=103)$ and fourth-year psychology students of the Faculty of Psychology, National Pedagogical Dragomanov University $(n=60)$.

First, using the content analysis, the authors studied the types, specific nature and skills necessary for psychologists to perform the professional activity in the media space effectively. The obstacles which impede experts to communicate adequately with clients also were under consideration. At the next stage, the authors established a level of communicative competence of practicing psychologists and psychology students as well as a level of their self-control when interacting by means of V. Riakhovskyi Test and M. Snyder Test. Using Ch. Spielberger inventory, the authors studied trait and state anxiety of students and practicing psychologists. 


\section{Findings}

Survey results show that practicing psychologists perform three types of activities in the media space: educational, advertising (SMM) and counseling. The authors assessed the level of psychologists' involvement in the media space on the basis of available media services (Facebook, Instagram, YouTube, webinars) (Figure 1)

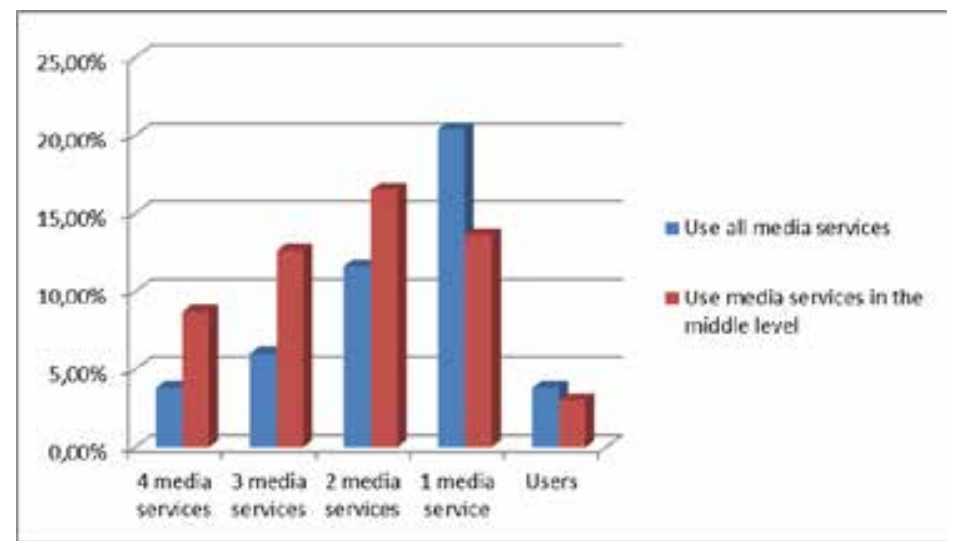

\section{Figure 1. The level of psychologists' involvement in the media space (\%)}

Taking into account the diagram, it is concluded as follows.

Only $3.8 \%$ (4 people) of respondents actively use all four media services: they create posts on Facebook and Instagram, stream lives and post stories, shoot YouTube videos, and hold webinars. 8.7\% (9 people) of interviewees use 4 media services but averagely, that is, their activity is irregular. For example, a practical psychologist dynamically keeps Instagram, constantly holds webinars and uploads video to YouTube-channel but rarely uses Facebook, or actively works at Facebook and Instagram and sometimes holds webinars or uploads videos to YouTube channel.

$6 \%$ (6 people) actively use 3 media services and $12.6 \%$ (13 people) use 3 media services averagely. The authors noticed a trend towards active use of Facebook and Instagram and less frequent carrying of webinars and uploading of videos to YouTube channel. 
$\mathbf{1 3 . 6 \%}$ (12 people) use 2 media services in their professional activity, and $16.5 \%$ (17 people) use 2 media services on average. The authors established that the respondents prefer to work on Facebook and Instagram, and they do not hold webinars at all and do not have their own YouTube channels.

$20.4 \%$ (21 people) use only 1 media service, and $13.6 \%$ (14 people) use only 1 media service at the average. Such respondents favour Facebook or Instagram depending on their target audience.

$3.8 \%$ (4 people) are active media users, and only $3 \%$ ( 3 people) use media services at the average. The peculiarity of media activity of the respondents is they are users of the information space, and they do not produce their own content but just receive the information necessary for their professional activity.

At the next stage, the authors studied the level and quality of involvement of practicing psychologists in the media activity using the example of Facebook. The findings are illustrated in Figure 2.

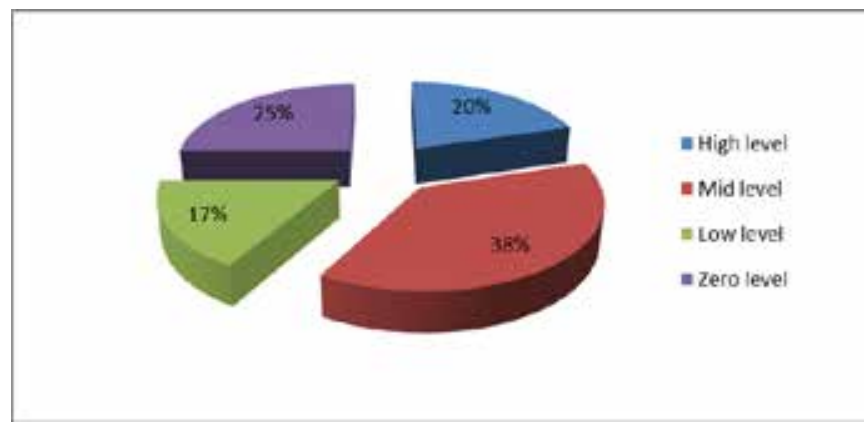

Figure 2. The level of the professional activity of psychologists in the media space (\%)

After analyzing the data, the authors obtained the following results.

$\mathbf{2 0 . 3 \%}$ (21 people) showed a high level of professional activity in the media space. The activities of such experts in the web space are distinguished by the publication of posts of informational, informational-motivational and personal character (from 5 posts per week); regular live streams; large number of followers (more than 1000 people, except 5000 friends); availability of a business page; active content commenting (20 or more 
comments on each post); a lot of likes $(30-250)$. These psychologists have well-formed content, own specialization, conduct marathons, trainings, online conferences on Facebook. 38\% (39 people) showed moderate activity. Their online activities consist of publications of posts of informational, informational-motivational and personal character (up to 5 posts per week); live streams are not regular; they don't have followers (up to 5000 friends); a business page is not always available; the number of comments is much lower (up to 10 to every post) as well as the number of likes (up to 20). These psychologists publish new content irregularly and occasionally. It is diverse, informative, and personal posts usually prevail over the information and motivational ones. Such experts rarely run marathons and take hold online events, and they don't have experience in creating online courses.

$16.7 \%$ (17 people) showed a low level of professional activity in the media space. As a rule, they publish posts of purely informative and personal character (up to 5 posts per month); they don't perform live streams; they don't have followers (up to 1000 friends); a lack of business page; the number of comments is much lower (up to 5 for each post); the number of likes is also small (up to 10). Such psychologists almost do not present and promote their professional activity on the network, and sometimes they share professional-information content. Their content is occasional and covers a small number of followers.

$25 \%$ ( 26 people) have zero activity - they do not demonstrate their professional activity on the social network but use it exclusively for personal use.

The authors also found that active users (58.3\% of respondents) can be conditionally divided into three groups: those who actively use text content; those who actively use video content; those who actively use both video and text content (Figure 3).

Based on the diagram, it is obvious that $61.6 \%$ (37 people) mostly use text content on the social network. Such psychologists publish texts of informational, informational-motivational and personal nature using their own photos or pictures. In their posts, they cover various developments in psychology, invite to counseling and recommend their services, offline and online activities and share achievements and events in their lives.

$\mathbf{1 8 . 8 \%}$ (11 people) actively run live streams and shoot videos. Their content is usually informational and motivational, sometimes personal. In their videos, such psychologists typically share their own successes, invite 


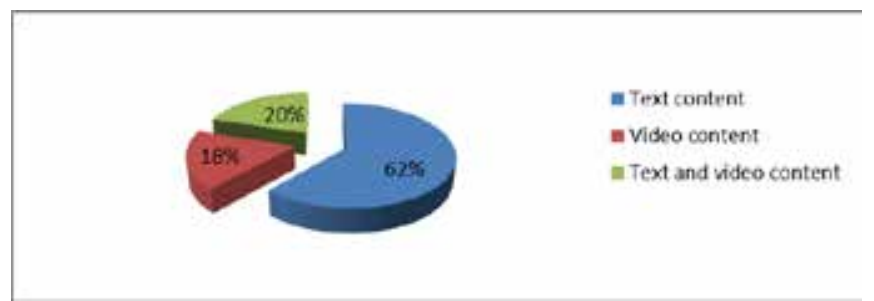

Figure 3. Quantitative indicators of the use of certain types of professional content in the media space (\%)

to various events, promote the services of their colleagues and own products, conduct marathons and online trainings.

$20 \%$ (20 people) actively write posts, run live streams and shoot videos. In their publications they promote own products and consultations, interesting events in psychology, conduct marathons and online training.

At the next stage, the authors examined the level of communication skills of practical psychologists and students psychologists using V.F. Ryakhovskiy Test $[1$, p. 554]. After analyzing the data, the authors obtained the following results (Table 1).

Table 1

The ratio of development level of communication skills of practical psychologists and psychology students (according to the method of V.F. Ryakhovskiy)

\begin{tabular}{|l|c|c|}
\hline \multicolumn{1}{|c|}{ Test results } & Practical psychologists & Psychology students \\
\hline Very low level & $0 \%$ & $0 \%$ \\
\hline Low level & $0 \%$ & $1,7 \%$ \\
\hline Passable level & $12,6 \%$ & $11,7 \%$ \\
\hline Medium level & $45,6 \%$ & $25 \%$ \\
\hline Sufficient level & $24,2 \%$ & $51,6 \%$ \\
\hline High level & $13,8 \%$ & $10 \%$ \\
\hline Very high level & $3,8 \%$ & $0 \%$ \\
\hline
\end{tabular}

For perception convenience and comprehension, the authors unite some levels and established the following: 
None of the practicing psychologists have a low level, but one psychol-

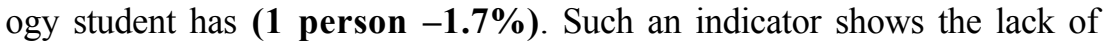
communication skills of this person. For the future psychologist, a low level of communication skills is not advisable because it reduces the expert's communicative competence and impedes professional activity. Sociability is one of the main features of the future psychologist that significantly influences the effectiveness of cooperation between the psychologist and the patient.

Medium level is peculiar to practicing psychologists (47 people 45.6\%) and psychology students (22 people $-36.7 \%)$. These people are intellectually curious, sufficiently patient in communication with others, they eagerly listen to an interesting interlocutor, assert their view without temper and they usually find it difficult to argue their views. It is difficult for such students to establish and maintain new contacts, that is, they do not have co-working skills.

Practicing psychologists (53 respondents $-\mathbf{4 2 . 8 \%}$ ) and psychology students $(37$ respondents $\mathbf{- 6 1 . 6 \% )}$ have a high level. Such persons are very sociable, tolerant in communication, they are easy to strike up an acquaintance with new people, form and express their opinions and argue it as well as curiously get into a discussion. Such students are well versed in verbal and non-verbal communication, and they have a high level of communication control. They know and apply the rules of ethics, adhere to the culture of verbal communication during conversation. These individuals are easy to speak to in public and are not ashamed of the cameras.

To evaluate the level of communicative control of psychology students and practicing psychologists, the authors used the assessment of self-control in communication (M. Snyder) [5, p. 558] (Figure 4).

Analyzing the data, the authors obtained the following results:

7 students $(11.7 \%)$ and 11 practicing psychologists $(10.6 \%)$ have a low level of communication control. These individuals find it difficult to control their rhetoric, and they behave inadequately and emotionally when communicating with friends, family, colleagues or clients. Students behave uncontrollably not only at lectures or seminars but also in everyday life. They do not control their rhetoric - both towards each other and towards older people - lecturers, parents, etc. That kind of behaviour is often associated with problems in education as well as with inadequate self-esteem. They quite often use swearwords in communication. 


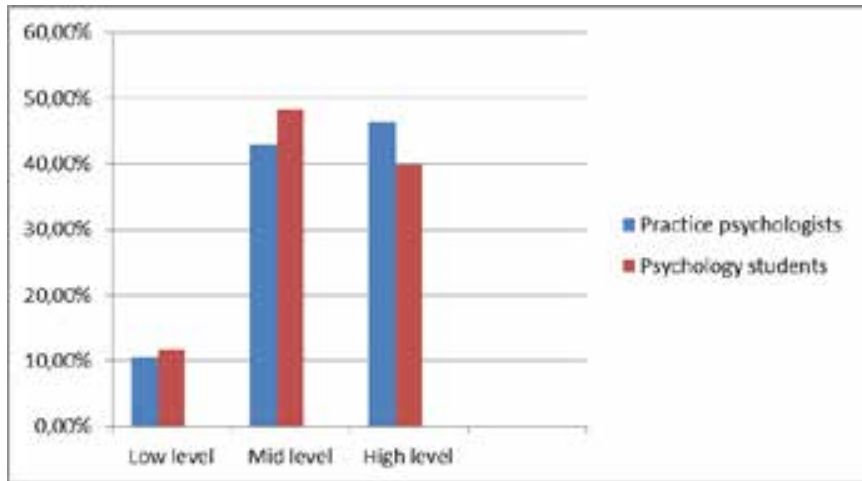

Figure 4. Correlation of development level of communication control of practicing psychologists and psychology students (according to M. Snyder method)

29 students (48.3\%) and 44 psychologists demonstrated a medium level of communication control. Such people are sincere but unrestrained in their emotional manifestations, and they are affected by a public opinion. Students quite easily take the side and the model of behaviour of a person who is respectable by them and has some influence. It is difficult for these students to control their communication, and they often become involved in discussions not always understanding their purpose and content. They frequently initiate conflicts or promote their escalation.

24 students $(40 \%)$ and 48 psychologists $(46.4 \%)$ have a high level of communication control. Such people easily adjust to any role and are flexible in responding to a change of situation, feel well and even can anticipate the impression they have made. These students are tactful - they control their speech and respectfully communicate with others. They have a high standard of ethics and rules of a culture of verbal communication. They are good in negotiating, thus, the students often act as peacemakers in conflict situations, help to make a compromise or resolve a conflict.

During the latter stage, the authors evaluated the levels of trait and state anxiety of students and practicing psychologists using Ch. Spielberger inventory [9, p. 58] (Figure 5). 


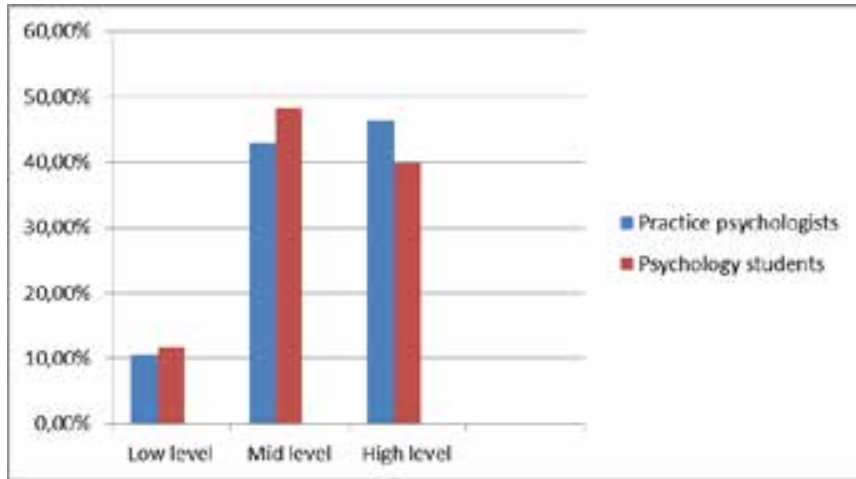

Figure 5. Indicators of state anxiety (SA) of practicing psychologists and psychology students (\%)

According to the diagram, it is obvious the following:

6 practicing psychologists $(5.8 \%)$ and 10 students (16.6\%) have a high level of state anxiety, 71 psychologists (69\%) and 39 students $(65 \%)$ have medium levels of state anxiety, and 26 psychologists $(\mathbf{2 5 . 2} \%)$ and 11 students $(\mathbf{1 8 . 4 \%})$ have a low level of state anxiety. This is a reminder that state anxiety is a state characterized by subjective experienced emotions: tension, worry, concern, nervousness.

The state is an emotional reaction to a stressful situation and may vary in intensity and dynamic over time. Psychologists who have demonstrated a high level of state anxiety have problems with public speaking, that is, they are afraid to speak for camera.

Students who have demonstrated a high level of state anxiety often and actively experience emotional tension, anxiety, fatigue, confusion in certain life situations (public speaking at a seminar, doing a test, passing a credit or an exam). The very state anxiety can lead to challenges in communication with people, during public speeches and, thus, reduce the psychologist's communicative competence. Such students have tension and excitement in the case public speech, which is the cause of non-expressive speech, text forgetting, loss of train of thoughts etc.

The authors also determined the level of personal anxiety and obtained the following results (Figure 6). 


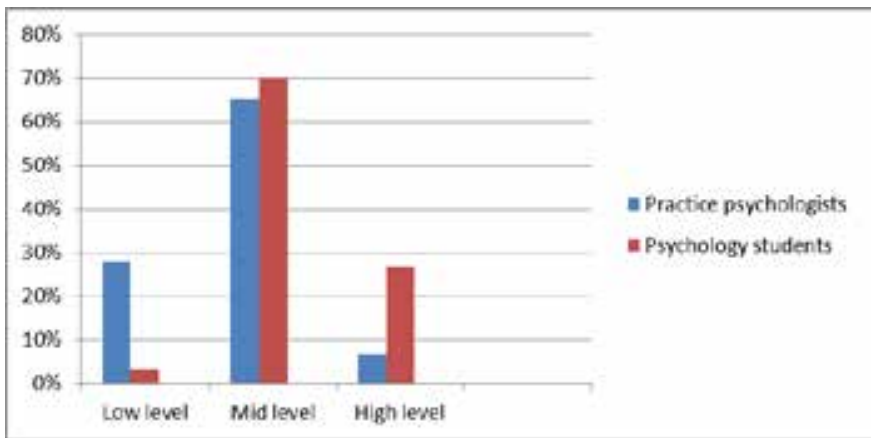

Figure 6. Indicators of personal anxiety (PA) of future psychologists and practicing psychologists (\%)

The diagram shows as follows:

7 psychologists (6.7\%) and 16 students (26.7\%) have a high level of personal anxiety, 67 psychologists $(65.3 \%)$ and 42 students $(70 \%)$ have a medium level of anxiety, and 29 psychologists (28\%) and only 2 students (3.3\%) have a low level of personal anxiety. The authors comprehend personal anxiety as persistent individual characteristics of the individual reflecting the subject's tendency to anxiety and assuming. It involves perception of a wide range of situations as threatening which lead to a specific reaction. Students who have a high level of personal anxiety are constantly experiencing concern, excitement, tension; they are often diffident and therefore, they are affected by the public opinion.

Applying the Pearson correlation coefficient, which is $r_{\mathrm{xy}}=1349 /(54.809 \times 35.86)=0.73183$ (for practicing psychologists) and $r_{x y}=1349 /(54.809 \times 35.86)=0.68652$ (for students), the authors proved that the level of personal anxiety of the interviewed group correlates with the level of communicative competence. The higher anxiety level is the lower communicative competence is.

Taking into account the fact that two indicators do not have a significant difference, the authors conclude that practical psychologists have undeveloped communicative competence since adolescence as well as a high level of state and personal anxiety. They have not worked through fears of public performance since the period studying at a university. 
Thus, it is evident that psychology students (47.4\%) and practicing psychologist $(45.6 \%)$ have a relatively low level of communication competence. Some psychology students $(16.6 \%)$, in contrast to practicing psychologists $(5.8 \%)$, have a high level of state anxiety. A state of personal anxiety has a similar situation: psychology students (26.7\%) and psychology practitioners $(6.7 \%)$. In view of the findings, it is concluded that it is necessary to form a high level of communicative competence and create relevant measures for the prevention of state and personal anxiety of future psychologists during the period of training at the university.

Thus, the authors established that nowadays, successful professional fulfillment of a practical psychologist needs the involvement of media tools in the professional activity which provide a high level of the development of communicative skills of a person. The paper confirms that a high level of anxiety adversely affects the level of communicative competence of practicing psychologists and students - future psychologists, which also influences the effectiveness and success of professional activity, in particular in the media space, and respectively professional self-realization of a psychology expert.

\section{Conclusions}

The findings show that it is essential to form relevant knowledge, expertise, skills and a high level of communicative competence of future experts according to the specific nature of a psychologist's activity in the media space. It is concluded that modern practicing psychologist should 1) be aware of available media tools and the specifics of their functioning; 2) be motivated to expand and modernize communication channels with clients; 3 ) be able to use media channels for educational and marketing (SMM) activities skillfully and appropriately; 4) should understand the specifics of online customer service and take it into account to improve their activities; 5) have a high level of communication competence for successful interaction with clients.

As the current market for psychological services requires the introduction of media services into professional activity, it is important to comprehend what kind of knowledge and skills of media future professional psychologists should have for their self-realization. Therefore, there is a need for the formation of specific professional communicative competence and the introduction of relevant disciplines and special courses in the process of training future psychologists. 


\section{References:}

1. Raygorodskiy D.Ya. (1998). Prakticheskaya psikhodiagnostika. Metodiki i testy. [Practical psychodiagnosis. Methods and tests]. Samara: BAKhRAKh.

2. Bonk C.J. (2009). The World is Open: How Web Technology Is Revolutionizing Education. LearnTeachLib (electronic journal), vol. 7, no. 2, pp. 3371-3380. Retrieved from: https://www.learntechlib.org/primary/p/31963/ (accessed 7 Augest 2019).

3. Giles D. (2003). Media psychology. New Jersey: LAWRENCE ERLBAUM ASSOCIATES PUBLISHERS.

4. Gist R., Stolz, S. (1982). Mental health promotion and the media: Community response to the Kansas City hotel disaster. American Psychologist, vol. 37, no. 10, pp. 1136-1139.

5. Mangold W.G. (2009). Social media: The new hybrid element of the promotion mix. Business Horizons (electronic journal), vol. 52, no. 4, pp. 357-365. Retrieved from: https://www.sciencedirect.com/science/article/pii/ S0007681309000329?via\%3Dihub (accessed 23 June 2019).

6. McCall R.B., Stocking S.H. (1982). Between scientists and public: Communicating psychological research through the mass media. American Psychologist, vol. 37, no. 9, pp. 985-995.

7. Pikas B. (2014). The Effectiveness of Online Advertising: Consumer's Perceptions of Ads on Facebook, Twitter and YouTubecope. Journal of Applied Business and Economics (electronic journal), vol. 16, no. 4, pp. 70-81. Retrieved from: http://digitalcommons.www.nabusinesspress.com/JABE/PikasB_Web16_4_.pdf (accessed 10 June 2019).

8. Rickheit G. (2008). Handbook of Communicative Competence. Gottingen : Hubert \& Co.

9. Spielberger C.D. (1972a). Anxiety as an Emotional State. In C. D. Spielberger (Ed.), Anxiety: Current Trends in Theory and Research. New York: Academic Press.

10. Wallace P. (2005). The psychology of the Internet. Maryland: Cambridge University Press. 